\title{
Susceptibilidade magnética e a distribuição de tamanhos das junções intergranulares em supercondutores policristalinos $\mathrm{Hg}, \mathrm{Re}-1223$
}

Bolzan, M. S. ${ }^{1}$

1 Programa de Pós-Graduação em Ensino de Física, Instituto Federal do Espírito Santo, Cariacica, ES, Brasil.

*e-mail: marcioifes@gmail.com

\begin{abstract}
Resumo
A fim de se entender melhor as propriedades microestruturais de cerâmicas policristalinas supercondutoras, avaliou-se que é possível estabelecer uma correlação direta entre a susceptibilidade magnética AC e a distribuição de tamanhos de junção intergranulares presentes numa amostra policristalina do supercondutor $\mathrm{Hg}, \mathrm{Re}-1223$. Para tanto essa estrutura policristalina foi descrita por uma Rede de Voronoi 2D e, recuperados os conceitos fundamentais para a descrição da resposta magnética da rede, foi possível mostrar que para cada campo magnético aplicado existe um conjunto de junções com mesmo tamanho que responde simultaneamente a este campo aplicado, sendo nessas condições momentaneamente, tais junções, as responsáveis pela resposta magnética da amostra. Essa descrição pode ser estendida a fim de demostrarmos que a derivada em primeira ordem da susceptibilidade magnética $A C$ em respeito ao campo magnético aplicado pode oferecer a forma, qualitativa, da distribuição estatística de tamanhos de junções na amostra analisada.
\end{abstract}

\begin{abstract}
In order to better understand the microstructural properties of polycrystalline ceramic superconductors, assessed that it is possible to establish a direct correlation between the magnetic susceptibility AC and distribution of intergranular junction sizes present in a polycrystalline sample of superconductor $\mathrm{Hg}, \mathrm{Re}-1223$. To achieve this polycrystalline structure was described for a 2D Voronoi Network, and recovered the fundamental concepts for the description of the magnetic response from the network, it was possible to show that for each applied magnetic field is a set of junctions with the same size which responds concurrently to this applied field, and these conditions momentarily, such junctions are responsible for the magnetic response of the sample. This description can be extended for to demonstrate that the first order derivative of the AC magnetic susceptibility with respect to the applied magnetic field can offer the form qualitative, the statistical distribution of sizes of junctions in the sample.
\end{abstract}

Keywords (Palavras chaves): propriedades magnéticas, cerâmicas policristalinas, hg,re-1223

\section{Introdução}

As bordas dos grãos, que formam as junções em amostras cerâmicas, são regiões que apresentam estequiometria diversa das regiões intragranulares. Essas regiões apresentam muito mais defeitos estruturais que a região intragranular, portanto também possuem propriedades supercondutoras diferentes das do interior do grão. No caso do supercondutor $\mathrm{Hg}, \mathrm{Re}-1223$, esses parâmetros foram estudados em detalhes, sendo que além da morfologia e composição, essas junções foram classificadas como isolantes [1].

Além de correntes de blindagem intragranulares, uma amostra supercondutora policristalina na presença de campo magnético aplicado apresentará correntes de blindagem intergranulares, se existirem junções entre grãos.

A eficiência da blindagem magnética, por conta das correntes intergranulares, é inversamente proporcional ao campo magnético aplicado. Portanto espera-se que a intensidade máxima dessas correntes seja limitada em função do fluxo magnético em torno das junções que transportam tais correntes. Ao transpor uma barreira essas correntes de blindagem, por serem governadas pelo mecanismo Josephson de transporte, obedecem um padrão de difração tipo Franhoufer na presença de campo aplicado [2]. Portanto seu comportamento segue a expressão 


$$
J_{c}(H)=\frac{J(0) \phi_{0}}{\pi \phi}\left|\sin \left(\frac{\pi \phi}{\phi_{0}}\right)\right|
$$

onde $\phi$ é fluxo de campo magnético em torno da junção e $\phi_{0}=2,0678 \cdot 10^{-15} \mathrm{~Wb}$ o quantum de fluxo.

Neste trabalho apresentamos uma descrição para a resposta magnética da amostra policristalina na presença de campo magnético baseada na consolidação de circuitos de corrente blindantes mapeadas pela expressão (1). Estendemos essa descrição a fim de recuperar informações quanto às características microestruturais da amostra e apresentamos uma interpretação que permite avaliar, a priori, uma descrição qualitativa da distribuição de tamanho de junções intergranulares presentes na amostra.

\section{Medidas de Susceptibilidade Magnética AC}

\subsection{Aparato experimental}

As medidas de suscetibilidade realizadas em laboratório utilizam um método de avaliação de tensões em bobina. A bobina externa gera um campo magnético alternado nas duas bobinas internas. Estas bobinas internas são iguais, entretanto uma é enrolada em sentido contrário ao da outra de modo que a diferença de potencial induzido $V_{b}$ na segunda bobina seja igual, porém de sinal trocado a diferença de potencial induzido $V_{a}$ da primeira bobina. O dispositivo Lock-in (amplificador para medir sinais alternados de pequena intensidade, com detecção de fase e filtros finos de frequência) conectado às bobinas internas mede a diferença de potencial entre elas. A diferença de potencial detectada no Lock-in deve ser nula. No entanto, se um material com propriedades magnéticas for colocado dentro de uma das bobinas, a diferença de potencial detectada pelo Lock-in não será.

\subsection{Dados obtidos}

A Figura 1 apresenta resultados obtidos para a parte real da susceptibilidade em função do campo aplicado para uma amostra em pastilha do supercondutor $\mathrm{Hg}, \mathrm{Re}-1223$. Sabe-se que amostras sintetizadas em pastilhas apresentam um degrau a mais na susceptibilidade em função da maior eficiência de blindagem por conta das correntes intergranulares presentes [3]. Estas medidas foram obtidas a $77 \mathrm{~K} \mathrm{e}$ indicam o primeiro campo crítico do grão $\left(H_{c 1}^{g}\right)$ em 40 $\mathrm{mT}$.

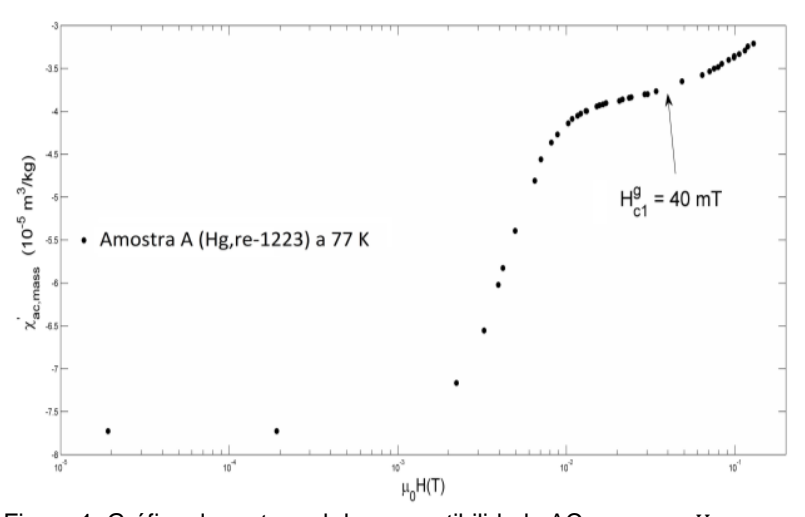

Figura 1: Gráfico da parte real da susceptibilidade AC versus $\mu_{0} H$ numa amostra supercondutora em pastilha da cerâmica $\mathrm{Hg}, \mathrm{Re}-1223$ a $10 \%$ $\mathrm{PO}_{2}$ em $77 \mathrm{~K}$.

\section{Distribuição de tamanhos da junções em amostras policristalinas}

A expressão (1) nos mostra que a medida que aumentamos o campo magnético aplicado, menor é corrente que passa pela junção. Consequentemente, uma vez definido um circuito de blindagem, se a corrente que passa pelas junções é reduzida, a eficiência da blindagem magnética também será reduzida, o que, consequentemente, diminui o módulo de $\chi^{\prime}$. Este efeito da redução da corrente que passa as junções com o incremento do campo magnético é o responsável pela perda de eficiência na blindagem magnética da amostra cerâmica policristalina.
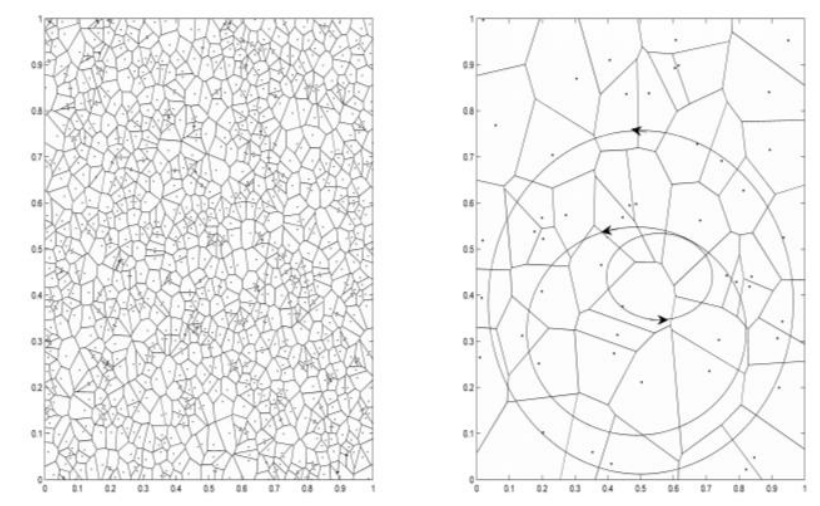

Figura 2: Representação esquemática das correntes de blindagem intergranular.

A amostra cerâmica policristalina de HgRe-1223 analisada aqui apresenta junções com morfologia retangular semelhante ao apresentado na Figura 2, onde a morfologia da amostra foi descrito pela rede de Voronoi 2D [4], onde $L$ representa o tamanho da junção, perpendicular ao campo, e $d$ a espessura isolante da junção, perpendicular ao campo e ao tamanho da junção. A corrente que passa através dessa junção se comporta segundo o padrão Franhoufer descrito na expressão (1). O fluxo magnético calculado em torno da junção será, numa aproximação de London, 


$$
\phi=\mu_{0} H L\left(d+2 \lambda_{a b}\right) .
$$

Inserindo (2) em (1) obtém-se

$$
J(H)=\frac{J(0) \phi_{0}}{\pi \mu_{0} H L\left(d+2 \lambda_{a b}\right)}\left|\sin \left[\frac{\pi \mu_{0} H L\left(d+2 \lambda_{a b}\right)}{\phi_{0}}\right]\right| .
$$

Considerando que essa espessura magnética da junção $t_{b}=d+2 \lambda_{a b}$ varia muito pouco de junção para junção, visto que $\lambda_{a b}$ e $d$ também variam muito pouco, a expressão (3) indica que, para um mesmo campo magnético aplicado, junções com tamanhos $L$ diferentes apresentaram comportamento de máximos e mínimos diferentes. Isso pode ser observado na Figura 3, onde obtemos o comportamento de junções com tamanhos diferentes, numa mesma faixa de variação de campos magnético aplicado. É importante lembrar que $J(0)$ depende da densidade de portadores de carga presentes nas junções e do acoplamento entre as fases das funções de ondas dos ensembles de super-elétrons nos supercondutores que formam uma junção. No caso estudado aqui, esses parâmetros (densidade de portadores e acoplamento de fases) variam muito pouco de grão para grão, visto que os grãos são todos de um mesmo supercondutor, podendo somente variar, muito pouco, em função de defeitos específicos não gerais para a amostra.
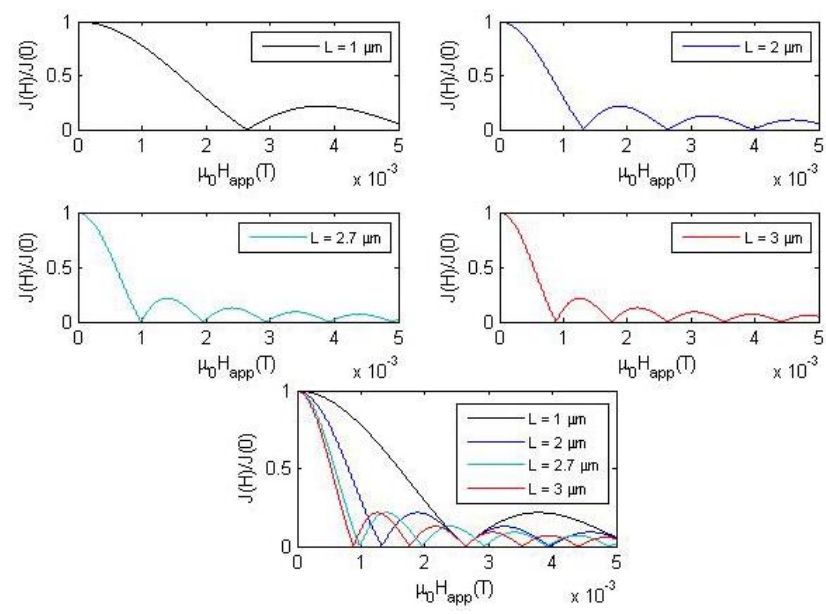

Figura 3: Simulação do comportamento de $J_{c}(H)$ numa junção retangular com $t_{b}(0,77 K)=372 \mathrm{~nm}$.

Para essas simulações utilizou-se $t_{b}=372 \mathrm{~nm}$ à $77 \mathrm{~K}$, onde $d$ foi assumido como da ordem de $2 \mathrm{~nm}$ e $\lambda_{a b}$ aproximadamente $185 \mathrm{~nm}$ [5]. O tamanho médio das junções, medido através da técnica de SEM para a amostra de $\mathrm{Hg}, \mathrm{Re}-1223$ analisada, foi de $2700 \mathrm{~nm}$ [1].

\section{Conclusões}

Pode-se observar através da expressão (3) que junções com comprimento $\boldsymbol{L}$ pequeno tem seu primeiro ponto de zero de corrente associado a fluxos magnéticos mais elevados. De forma contrária, junções com $L$ de maior comprimento tem seu primeiro ponto de zero de corrente associado com menores valores de campo magnético aplicado. O comportamento apresentado na Figura 1 se deve ao fato de que para cada valor de campo um certo número de junções atingem seu primeiro mínimo. Esse comportamento faz com que as correntes de blindagem que passam por essas junções tendam a zero, consequentemente contribuindo para com a diminuição do módulo de $\chi^{\prime}$.

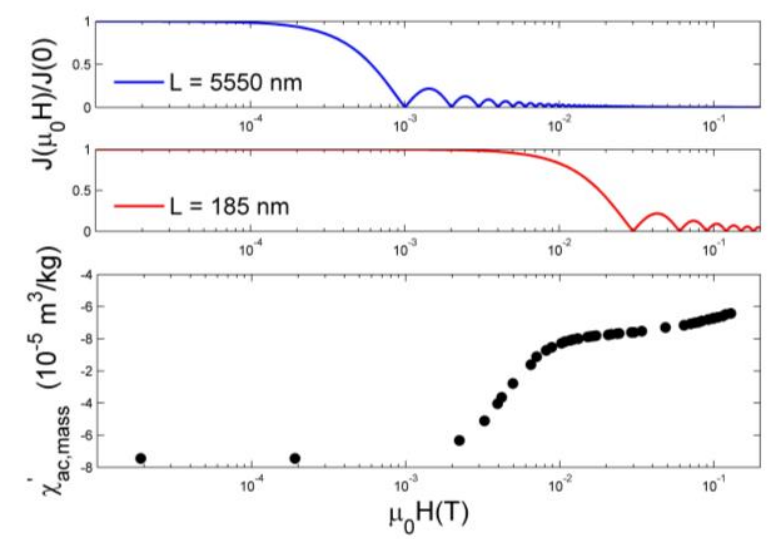

Figura 4: Simulação do comportamento de $J_{c}(H)$ numa junção retangular com $t_{b}(0,77 \mathrm{~K})=372 \mathrm{~nm}$ para obter os tamanhos máximos e mínimos das junções da amostra $\mathrm{Hg}, \operatorname{Re}-1223$ através do diagrama de $\chi^{\prime}(H)$.

A expressão (1) descreve que o parâmetro relevante neste modelo é o fluxo de campo magnético. Considerando o campo magnético fixo, o fluxo magnético será definido pelo produto de $\boldsymbol{t}_{\boldsymbol{b}}$ e $\boldsymbol{L}$, ou seja, uma área efetiva de fluxo em torno da junção, $\boldsymbol{A}_{\boldsymbol{e f f}}$. As junções que possuem mesma área efetiva de fluxo, $\boldsymbol{A}_{\boldsymbol{e f f}}$, tem seus mínimos coincidindo para um mesmo valor de campo magnético sucessivamente.

Pode-se estimar o tamanho das junções que estão atingindo seu primeiro zero para um determinado campo, caso consideremos $t b$ como um valor médio fixo. A Figura 4 indica os tamanhos máximo e mínimo das junções que compõem a amostra de Hg,Re-1223 submetida às medidas de susceptibilidade. Verifica-se que para que a amostra se comporte conforme apresentado na Figura 1 é necessário ter junções com tamanhos $L$ variando entre $185 \mathrm{~nm}$ e $5550 \mathrm{~nm}$.

Uma análise mais aprofundada da Figura 3 indica que para um valor específico de campo magnético tem-se somente um número definido de junções com mesma $A_{\text {eff }}$ que podem atingir seu zero de corrente. Portanto, pode-se concluir que quanto maior for o número de junções com determinada área $A_{\text {eff }}$, maior será a contribuição desse tipo de junção para a variação de $\chi^{\prime}$ para um determinado valor de campo magnético. Mais ainda, analisando a questão sob outro ponto, é possível interpretar que a derivada de primeira ordem da susceptibilidade magnética $\chi^{\prime}$ em relação ao campo magnético aplicado, deve fornecer uma boa aproximação para o tipo de distribuição de áreas Aeff das junções presentes na amostra em análise. Essa curva é apresentada na Figura 5 e pode ser associada a 
uma distribuição do tipo Gamma. Assumindo tb como um valor invariante em média de junção para junção, a diferença de Aeff de junção para junção está associada a $L$, ou seja, a distribuição Gamma proposta é oriunda da distribuição de $L$ da amostra. Em resumo, o tamanho $L$ das junções pode ser descrito estatisticamente segundo uma distribuição do tipo Gamma, como já proposto empiricamente na literatura [1].

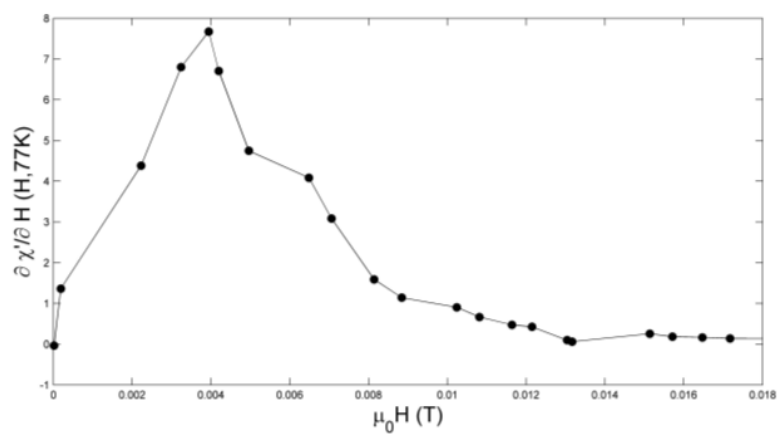

Figura 5: O gráfico da derivada de primeira ordem de $\chi^{\prime}(H)$ com respeito a $H$ obtido a partir das medidas apresentadas na Figura 1.

É importante enfatizar a similaridade da interpretação proposta para bulks poliscristalinos com a mesma construção estendida para filmes espessos. Sem qualquer perda de generalidade, a construção morfológica e estrutural das junções é praticamente a mesma. No caso de filmes espessos a associação é quase imediata, basta comparar a Figura 2 com imagens SEM disponíveis na literatura [6].

\section{Agradecimentos}

O Autor agradece à FAPES pelo apoio financeiro através dos projetos 67653952/2014 e 71885633/2015.

\section{Referências}

\section{Artigos}

[1] PASsOS, C. A. C, et al., Phys. Rev. B, v. 74 p. 094514, 2006.

[3] SIN, A., et al., Phys. C, v. 328, p. 80, 1999.

[5] PANAGOPOULOS, C., et al., Phys. Rev. Lett., v. 79, p. 2320, 1997.

[6] SCHILLING, M., et al., J. Phys. D: Appl. Phys., v. 47, p. 034008, 2014.

\section{Capítulo de livro}

[2] PASSOS, C. A. C., et al. Superconductors, Editora INTECH, p. 377 - 394.

[4] BERG, M. Computacional Geometry, Editora Springer-Verlag, p. $147-163$. 